\title{
Efeitos de um programa de exercícios no desempenho de crianças nos testes de flexibilidade e impulsão vertical
}

\author{
Diogo Henrique Constantino Coledam ${ }^{1,2}$ \\ Gustavo Aires de Arruda ${ }^{1}$ \\ Arli Ramos de Oliveira ${ }^{1,2}$ \\ ${ }^{1}$ Grupo de Estudo e Pesquisa em Atividade Física e Saúde, Centro de Educação Física e Esporte, \\ Departamento de Ciências do Esporte, Universidade Estadual de Londrina, PR, Brasil \\ ${ }^{2}$ Programa de Pós-Graduação Associado em Educação Física, Universidade Estadual de Maringá e \\ Universidade Estadual de Londrina, PR, Brasil
}

\begin{abstract}
Resumo: Esse estudo investigou o efeito de um programa de exercícios na flexibilidade e impulsão vertical de escolares. 61 crianças (30 meninos) foram divididas em Grupo Controle Masculino (GCM), Grupo Intervenção Masculino (GIM), Grupo Controle Feminino (GCF) e Grupo Intervenção Feminino (GIF). O GIM e GIF foram submetidos a um programa de exercícios durante as aulas de Educação Física Escolar com duração de 12 semanas. Foram realizados os testes de "sentar-e-alcançar" e impulsão vertical anteriormente às 12 semanas e após o término deste programa. Os resultados indicaram que o GIF e o GIM aumentaram significativamente o desempenho nos testes de impulsão vertical e "sentar-ealcançar" após o programa de intervenção $(P<0,05)$. No GCM e GCF não foram verificadas diferenças significativas no desempenho do teste de impulsão vertical e "sentar-e-alcançar" $(P>0,05)$. O programa de intervenção utilizado nesse estudo foi eficiente em aumentar a flexibilidade e impulsão vertical de crianças.
\end{abstract}

Palavras-chave: Aptidão física. Alongamento. Escolares. Exercícios.

\section{Effects of an exercise program on children's flexibility and vertical jump performance}

\begin{abstract}
This study investigated the effects of an exercise training program on flexibility and vertical jump performance in children at scholar age. 61 Children (30 boys) were divided in male control group (MCG), male intervention group (MIG), female control group (FCG) and female intervention group (FIG). MIG and FIG were submitted to an exercise training program during physical education classes for 12 weeks. The "sit-and-reach" and vertical jump tests were performed before and after 12 weeks of the exercise training program. The results demonstrated that MIG and FIG groups significantly increased the performance on vertical jump as well as the "sit-and-reach" tests after the exercise training program $(P<0,05)$. There were no differences in MCG and FCG on vertical jump performance and "sit-and-reach" tests $(P>0,05)$. The exercise program used in this study was efficient to increase flexibility and vertical jump performance in children.
\end{abstract}

Keywords: Physical fitness. Stretching. School children. Exercises.

\section{Introdução}

No conceito de aptidão física relacionada à saúde e ao desempenho atlético observa-se o seguinte paradigma: a primeira engloba componentes que aparentemente podem oferecer alguma proteção quanto ao surgimento e desenvolvimento de disfunções degenerativas induzidas pelo estilo de vida sedentário (GUEDES, 2007; MEREDITH, 2008). $\mathrm{Na}$ segunda, os componentes que contribuem para um melhor desempenho esportivo, levando-se em consideração que cada especialidade esportiva tem exigências específicas (GUEDES, 2007). Neste paradigma, a flexibilidade é um componente da aptidão física relacionada à saúde e pode ser definida como a capacidade de realizar movimentos em determinadas articulações com amplitude de movimento adequada (BARBANTI, 2003). A potência muscular, definida como a propriedade de realizar esforços máximos no menor tempo possível, é um componente da aptidão física relacionada ao desempenho atlético (GUEDES, 2007).

A manutenção de parâmetros adequados de flexibilidade na região dos isquiotibiais parece ser um aspecto importante para prevenção de lombalgias. Dores na região lombar durante a adolescência podem apresentar taxas de prevalência de $17,2 \%$, podendo em alguns casos levar à incapacidade de realização das atividades 
normais, ou mesmo ao uso de medicamentos (FELDMAN et al., 2001). Além disso, há relação entre baixos parâmetros de elasticidade dos músculos isquiotibiais e lombares com dores lombares (JONES et al., 2005) e na cervical (MIKKELSSON et al., 2006).

Para avaliação dos níveis de flexibilidade, o teste de "sentar-e-alcançar" vem sendo bastante empregado, pois é um indicador da flexibilidade da coluna lombar (CHILLÓN et al., 2010) e dos músculos isquiotibiais (CASTRO-PIÑERO et al., 2009). Estudos demonstraram que em amostras representativas de crianças brasileiras, entre $42 \%$ e $49 \%$ dos meninos, e de $24 \%$ a $55 \%$ das meninas, não atendem os critérios de saúde estabelecidos para o teste de "sentar-e-alcançar" (DÓREA et al., 2008; RONQUE et al., 2007). O baixo desempenho em testes de aptidão física, que reflete em não atender os critérios de saúde adotados por diferentes baterias de testes (GUEDES, 2007) indica maior risco ao acometimento por problemas de saúde, no caso da flexibilidade dores na coluna vertebral (JONES et al., 2005; MIKKELSSON et al., 2006).

Apesar de a potência muscular ser classificada como componente direcionado ao desempenho atlético esta parece também estar associada com aspectos relevantes para a saúde (STEENE-JOHANNESSEN et al., 2009). Há uma relação inversa entre aptidão física determinada através de testes de potência aeróbia, potência muscular, resistência abdominal e lombar e fatores de risco metabólicos (STEENEJOHANNESSEN et al., 2009). Além disso, o desempenho de crianças em teste de potência muscular de membros inferiores é inversamente associado a marcadores inflamatórios, sendo que a associação aumenta em crianças com sobrepeso ( $\underline{R U I Z}$ et al., 2008). Além da saúde metabólica, a realização de exercícios de saltos parece aumentar a densidade mineral óssea (GUNTER et al., 2008; WEEKS et al., 2008). Para mensuração da potência muscular de membros inferiores, o teste de impulsão vertical tem sido muito utilizado em crianças submetidas à programas de exercício físico por ser sensível para avaliar o efeito do treinamento de força (CHANNELL; BARFIELD, 2008) e apresentar alta relação com a aceleração, agilidade e velocidade (PETERSON et al., 2006; VESCOVI; MCGUIGAN, 2008).

Diversos programas de intervenção realizados após o período normal de aula com o objetivo de aumentar a flexibilidade (BEETS; PITETTI, 2005; KOUTEDAKIS; BOUZIOTAS, 2003; SANTONJA et al., 2007; STEPHENS; WENTZ, 1998), impulsão vertical (BONHAUSER et al., 2005; STENEVI-LUNDGREN et al., 2009; 2010; WEEKS et al., 2008) e outras capacidades físicas (ARDOY et al., 2011) foram realizados. Por outro lado, poucas evidências estão disponíveis acerca do efeito de programas de intervenção realizados durante as aulas de Educação Física na escola. Levantamento realizado nas Bases de Dados Lilacs, Scielo, Sport Discus e Medline encontrou apenas dois estudos que analisaram o efeito de programas de intervenção por meio de exercícios durante as aulas de Educação Física sobre a flexibilidade (SAINZ DE BARANDA et al., 2006; RODRÍGUEZ et al., 2008) e um para impulsão vertical (WEEKS et al., 2008) em crianças.

A verificação da eficácia de programas de intervenção realizados durante as aulas de Educação Física escolar pode auxiliar os professores de Educação Física a planejarem suas aulas visando à melhora do desempenho motor de seus alunos, uma vez que apenas uma pequena parcela dos jovens é engajada em atividades esportivas ou programas de exercícios extra-escolares. Sendo assim, o objetivo desse estudo foi verificar o efeito de um programa de intervenção realizado por meio de exercícios durante as aulas de Educação Física escolar sobre a flexibilidade e impulsão vertical de crianças.

\section{Amostra}

\section{Materiais e Métodos}

Participaram do estudo 61 crianças (30 meninos e 31 meninas) regularmente matriculadas na rede municipal de ensino $\left(5^{\circ}\right.$ ano) de Nova Europa - SP. A amostra foi escolhida de forma intencional, e o critério de inclusão no estudo foi a não participação em qualquer tipo de treinamento físico sistematizado e a frequência de no mínimo $85 \%$ nas aulas de Educação Física. Os pais ou responsáveis pelos participantes assinaram um Termo de Consentimento Livre e Esclarecido de acordo com a Resolução no 196/96 especificando todos os procedimentos do estudo. $O$ projeto de pesquisa foi aprovado pelo Comitê de Ética e Pesquisa da UNESP - Universidade Estadual Paulista, protocolo ํㅜ 11017/46/01/10.

\section{Delineamento Experimental}

Os participantes foram divididos em quatro grupos, Grupo Controle Masculino (GCM) $n=15$, 
Grupo Intervenção Masculino (GIM) n=15, Grupo Controle Feminino (GCF) $n=15$ e Grupo Intervenção Feminino (GIF) $\mathrm{n}=16$. A idade, estatura, massa corporal e índice de massa corporal (IMC) da amostra são apresentados na Tabela 2.

Todos os procedimentos do estudo foram realizados na instituição a qual os alunos estavam matriculados. Todos os participantes foram orientados a manterem as atividades que estavam habituados a realizar. O GIF e GIM foram submetidos a um programa de intervenção realizado através de exercícios durante as aulas de Educação Física, que ocorriam duas vezes por semana com duração de 50 minutos. O GCF e GCM participaram das aulas de Educação Física normalmente, porém não foram submetidos ao programa de intervenção.

No início do programa de intervenção, foram realizadas medidas da massa corporal, por meio de uma balança digital com precisão de $100 \mathrm{~g}$, e a estatura foi verificada com um estadiômetro portátil fixado em uma parede com precisão de $0,1 \mathrm{~cm}$. Os testes de impulsão vertical e "sentare-alcançar" foram aplicados anteriormente às 12 semanas do programa de intervenção (PRÉ) e após o seu término (PÓS). Para a aprendizagem dos testes, todos os participantes receberam informações acerca dos procedimentos para a realização dos testes, observaram as demonstrações de ambos os testes, e então, foram submetidos a três tentativas de cada teste no mesmo dia. Os testes de "sentar-e-alcançar" e impulsão vertical foram escolhidos por representarem um componente da aptidão física relacionada à saúde (flexibilidade) e aptidão física relacionada ao desempenho atlético (potência muscular), respectivamente (GUEDES, 2007). Apesar do teste de impulsão vertical ser classificado como componente da aptidão física relacionada ao desempenho atlético, recentemente foi demonstrado que também está relacionado à saúde. O desempenho abaixo da média no teste de impulsão horizontal está associado à menor percepção de estado de saúde e menor satisfação com a vida e com relacionamentos familiares (PADILLA-MOLEDO et al., 2012). Além disso, o desempenho de crianças no teste de impulsão vertical é influenciado pelo estado nutricional, sendo os classificados como eutróficos obtendo maior desempenho comparado aos indivíduos classificados abaixo do peso e aos com sobrepeso (BOVET et al., 2007). Todas as medidas e testes foram realizados pelo mesmo pesquisador, no mesmo local, utilizando os mesmos equipamentos. Nas análises foi considerado o maior valor obtido nas três tentativas de cada teste. Segue a descrição da forma de aplicação dos testes:

Teste de impulsão vertical: Para a realização do teste de impulsão vertical foi fixada uma fita métrica de três metros em uma parede lisa. $O$ avaliado posicionava-se lateralmente a superfície graduada, com as plantas dos pés totalmente apoiadas sobre o solo, e com um braço completamente estendido acima da cabeça, onde foi marcado o ponto mais alto alcançado com o dedo médio. Para facilitar a marcação, foi utilizado pó de giz na extremidade dos dedos. A partir da posição ortostática, a execução consistiu em flexionar as pernas e executar a impulsão contra movimento, com auxílio dos braços, e tocar o ponto mais alto possível na parede. A marcação foi feita com precisão de $0,5 \mathrm{~cm}$. 0 valor foi calculado pela diferença da maior altura alcançada (com salto) e a altura parado, com os valores expressos em centímetros (GUEDES; GUEDES, 2006).

Teste de "sentar-e-alcançar": A flexibilidade foi avaliada através do teste de "sentar-ealcançar": utilizando um banco com dimensões de $30,5 \times 30,5 \times 30,5 \mathrm{~cm}$, com um prolongamento de $23 \mathrm{~cm}$ e uma fita graduada em milímetro. Para tanto, o indivíduo posicionava-se sentado com as pernas completamente estendidas, pés descalços, ligeiramente afastados e apoiados contra o banco. Foram realizadas três tentativas de flexão de tronco, empurrando a régua com a ponta dos dedos e mantendo os joelhos, cotovelos e punhos em extensão. $O$ avaliado era orientado a permanecer na posição máxima alcançada por três segundos, para a leitura da régua (GUEDES; GUEDES, 2006).

\section{Programa de intervenção por meio de exercícios}

O Programa de intervenção por meio de exercícios consistiu em exercícios utilizando cordas, que visavam o desenvolvimento da potência muscular, e exercícios de alongamento, com o objetivo de produzir melhora quanto à flexibilidade. O Programa de intervenção foi realizado durante as aulas de Educação Física, em uma quadra poliesportiva coberta. Ambos os tipos de exercícios foram organizados de forma 
que não interferissem no conteúdo da aula, de acordo com a proposta pedagógica da escola. Os grupos intervenção e controle de ambos os sexos realizaram as aulas de Educação Física com 0 mesmo conteúdo e objetivos. Durante o período do estudo, as aulas de Educação Física tiveram como objetivo a iniciação aos esportes individuais e coletivos por meio de jogos utilizando gestos de diferentes modalidades esportivas. A duração do programa de intervenção não ultrapassou 7 minutos por aula. Anteriormente ao início do Programa de intervenção todos os alunos foram informados acerca da ordem dos exercícios, tempo de execução de cada um e os materiais disponíveis para as atividades. Desta forma, os alunos se organizavam rapidamente.

Os exercícios de alongamento foram realizados ao final de cada aula, através do método de alongamento estático ativo e enfatizaram os grupamentos musculares dos membros inferiores. Os exercícios de alongamento realizados para cada grupamento muscular estão descritos no Quadro 1. A duração de cada exercício de alongamento ao longo das 12 semanas está descrita na tabela 1 .

Tabela 1. Duração dos exercícios de alongamento (ALO) e volume de saltos (SAL) ao longo das 12 semanas de intervenção.

\begin{tabular}{|c|c|c|c|c|c|c|c|c|c|c|c|c|}
\hline & 1 & 2 & 3 & 4 & 5 & 6 & 7 & 8 & 9 & 10 & 11 & 12 \\
\hline ALO & 10 & 10 & 10 & 15 & 15 & 15 & 20 & 20 & 20 & 25 & 25 & 25 \\
\hline SAL & 20 & 20 & 30 & 30 & 40 & 40 & $2 \times 30$ & $2 \times 30$ & $2 \times 40$ & $2 \times 40$ & $2 \times 40$ & $2 \times 40$ \\
\hline
\end{tabular}

ALO: Duração dos exercícios de alongamento (segundos); SAL: Quantidade de saltos (repetições).

Os exercícios utilizando cordas foram realizados no início de cada aula, ao término do aquecimento. O exercício foi realizado em trios, com dois participantes segurando a corda um em cada extremidade e o terceiro posicionado no ponto médio da corda. Ao sinal inicial, os dois participantes que tinham como objetivo segurar a corda, iniciavam o movimento de giro, obrigando o participante que estava no meio da corda saltar para que esta não tocasse em seus pés. A frequência de saltos foi estabelecida em 60 saltos por minuto, ao final o participante que havia terminado a quantidade determinada de saltos trocava de posição com um dos participantes que estava segurando a corda. Os alunos foram orientados a saltar sempre com ambas as pernas. O volume de saltos utilizados ao longo das 12 semanas está descrito na tabela 1. Os materiais utilizados foram 30 colchonetes e 10 cordas de nylon com $5 \mathrm{~m}$ de comprimento. Em cada sessão, um pesquisador acompanhou a intervenção, corrigiu e orientou os alunos.

Quadro 1. Descrição dos exercícios de alongamento.

\section{Grupamento muscular}

\section{Adutores}

\section{Gastrocnêmios}

3. Isquiotibiais

4. Isquiotibiais

5. Quadríceps

\section{Descrição do Exercício}

Após o afastamento lateral das pernas, o avaliado flexionou o joelho direito sem que este ultrapassasse os dedos do pé e manteve a perna esquerda estendida. $O$ exercício foi realizado para ambos hemicorpos.

Em pé com afastamento anteroposterior dos pés, o avaliado manteve a planta do pé de trás totalmente no chão enquanto empurrava uma parede com as mãos. 0 exercício foi realizado para ambos hemicorpos.

Sentado, com joelhos estendidos, o avaliado flexionou o tronco e alcançou o ponto mais distante possível na direção dos pés.

Sentado, com um joelho estendido e outro flexionado, o avaliado alcançou o ponto mais distante em direção ao pé da perna estendida. O exercício foi realizado para ambos hemicorpos.

Decúbito lateral, o avaliado flexionava o joelho direito mantendo-o junto ao corpo e segurava o tornozelo do mesmo pé. O exercício foi realizado para ambos hemicorpos.

\section{Análise Estatística}

O tamanho amostral foi calculado baseado nos resultados de dois estudos anteriores (ARDOY et al., 2011; DUNCAN; WOODFIELD, 2006), considerando diferença média de 3,0 e
3,2, desvio padrão de 3,1 e $4,0 \mathrm{~cm}$, tamanho de efeito de 0,90 e 0,80 para a flexibilidade e impulsão vertical respectivamente, utilizando o software G*Power 3.0.10 (Instituto de Psicologia, Dusseldorf, Alemanha). Desta forma, um número 
mínimo de 15 sujeitos por grupo foi considerado necessário para detectar possíveis diferenças significativas entre os momentos. O Teste de Shapiro-Wilk foi utilizado para análise da normalidade e a homogeneidade da variância foi verificada pelo teste de Levene. Os resultados foram descritos por meio de média e desviopadrão. Foi utilizada a análise de variância fatorial $(2 \times 2 \times 2)$ com medidas repetidas (ANOVA) tendo como variáveis independentes 0 grupo (intervenção $x$ controle), sexo (meninos $x$ meninas) e momento (pré $x$ pós). $O$ teste PostHoc de Tukey para "n" desiguais foi utilizado para indicação das interações. As variáveis dependentes foram os resultados do teste de "sentar-e-alcançar" e de impulsão vertical. A ANOVA One-Way foi utilizada para comparação da idade e características morfológicas dos grupos no momento inicial. O Delta percentual $(\Delta \%)$, a diferença média entre os momentos e os respectivos intervalos de confiança de $95 \%$ foram calculados para as variáveis dependentes em todos os grupos. A significância adotada foi de $5 \%$.

\section{Resultados}

Os grupos não apresentaram diferença significativa para idade, estatura, massa corporal e IMC (Tabela 2).

Tabela 2. Média (desvio-padrão) da idade, estatura, massa corporal e IMC dos participantes.

\begin{tabular}{lcccc}
\hline & \multicolumn{3}{c}{ Meninos } & \multicolumn{2}{c}{ Meninas } \\
\cline { 2 - 5 } & $\mathrm{GCM}(\mathrm{n}=15)$ & $\mathrm{GIM}(\mathrm{n}=15)$ & $\mathrm{GCF}(\mathrm{n}=15)$ & $\mathrm{GIF}(\mathrm{n}=16)$ \\
\cline { 2 - 5 } Idade (anos) & $9,67(0,72)$ & $9,47(0,64)$ & $9,33(0,49)$ & $9,5(0,63)$ \\
Estatura $(\mathrm{m})$ & $1,31(0,06)$ & $1,37(0,07)$ & $1,32(0,08)$ & $1,30(0,06)$ \\
Massa Corporal (kg) & $35,84(6,01)$ & $34,58(4,80)$ & $33,40(7,65)$ & $33,69(8,65)$ \\
$\mathrm{IMC}\left(\mathrm{kg} / \mathrm{m}^{2}\right)$ & $20,78(2,04)$ & $18,39(1,80)$ & $18,99(3,23)$ & $19,72(3,75)$ \\
\hline
\end{tabular}

$\mathrm{GCM}=$ Grupo controle masculino; GIM = Grupo de intervenção masculino; $\mathrm{GCM}=$ Grupo controle feminino; GIF= Grupo intervenção feminino e IMC = índice de massa corpórea.

Os resultados dos meninos e das meninas nos testes de impulsão vertical e "sentar-e-alcançar" nos momentos PRÉ e PÓS são apresentados na Tabela 3. O GIM e o GIF aumentaram significativamente o desempenho nos testes de impulsão vertical e "sentar e alcançar" após o programa de intervenção $(P<0,05)$. Para o GCM e GCF não ocorreram diferenças significativas entre os momentos PRÉ e PÓS $(P>0,05)$. Não foram encontradas diferenças inter grupos nos momentos PRÉ e PÓS. O delta percentual no GIF para o teste de impulsão vertical e "sentar-e- alcançar" foi maior em relação ao GIM. No Teste de "sentar-e-alcançar" houve um $\Delta \%$ negativo no GCM, o mesmo não ocorrendo com o GCF (tabela 3).

A diferença média e os respectivos intervalos de confiança de $95 \%$ entre os momentos PRÉ e PÓS foram de: $\mathrm{GIM}=4,57(2,74-6,05)$ e 2,40 $(0,82-3,97), \mathrm{GIF}=4,97(2,91-6,41)$ e $3,22(1,70-$ $5,02), \mathrm{GCM}=-0,14(-2,19-1,92)$ e $-1,23(-2,99-$ $0,52)$, GCF $0,70(-1,17-2,57)$ e $0,66(-0,85-$ $2,18) \mathrm{cm}$ para os testes de impulsão vertical e "sentar-e-alcançar" respectivamente.

Tabela 3. Resultados das meninas (GIF e GCF) e dos meninos (GIM e GCM) nos Testes de "Sentare-Alcançar" (SA) e Impulsão Vertical (IV) nos momentos antes (PRE) e após (PÓS) 12 semanas do programa de intervenção.

\begin{tabular}{lcccccc}
\hline \multirow{2}{*}{$\begin{array}{c}\text { Grupo } \\
\text { Momento }\end{array}$} & \multicolumn{2}{c}{ GIM } & \multicolumn{5}{c}{ GCM } \\
\cline { 2 - 7 } & PRÉ & PÓS & $\Delta \%$ & PRÉ & PÓS & $\Delta \%$ \\
\cline { 2 - 7 } SA (cm) & $(\mathrm{n}=15)$ & $(\mathrm{n}=15)$ & $(\mathrm{n}=15)$ & $(\mathrm{n}=15)$ & \\
IV (cm) & $24,03(5,61)$ & $26,43(6,89) \dagger$ & 9,99 & $25,43(4,84)$ & $24,20(6,73)$ & $-4,84$ \\
& $23,73(8,52)$ & $28,30(6,96)^{*}$ & 19,26 & $23,97(5,10)$ & $23,83(5,47)$ & $-0,58$ \\
\cline { 2 - 7 } & \multicolumn{7}{c}{ GIF } & $(\mathrm{n}=16)$ & $(\mathrm{n}=15)$ & $(\mathrm{n}=15)$ \\
SA (cm) & $24,72(6,73)$ & $27,94(6,30)^{*}$ & 13,03 & $25,53(6,09)$ & $26,20(5,58)$ & 2,62 \\
IV (cm) & $22,19(5,23)$ & $27,16(5,12)^{*}$ & 22,40 & $26,50(6,13)$ & $27,20(6,85)$ & 2,64 \\
\hline
\end{tabular}

Resultados apresentados em média (desvio padrão); * $P<0,001 ; \dagger P<0,05$; $G C M=$ Grupo controle masculino; $\mathrm{GIM}=$ Grupo de intervenção masculino; $\mathrm{GCM}=$ Grupo controle feminino; GIF= Grupo intervenção feminino, SA = resultado do teste de "sentar e alcançar" e IV = resultado do teste de impulsão vertical. 


\section{Discussão}

O objetivo do presente estudo foi verificar o efeito de um programa de intervenção realizado por meio de exercícios sobre a flexibilidade e impulsão vertical de crianças escolares. Os resultados demonstraram que o programa de intervenção aumentou significativamente 0 desempenho das crianças nos testes de "sentare-alcançar" e impulsão vertical no GIF e GIM, enquanto que no GFC e GMC não ocorreram diferenças significativas.

Os resultados do teste de "sentar-e-alcançar" do presente estudo corroboram com outros que demonstraram aumento significativo da flexibilidade após programas de intervenção realizados durante as aulas de Educação Física que consistiram de $5 \mathrm{~min}$. de alongamentos para os músculos isquiotibiais, com frequência de duas vezes na semana (SAINZ DE BARANDA et al., 2006; RODRÍGUES et al., 2008). Desta forma, evidencia-se a possibilidade de aumentar a flexibilidade de crianças por meio de pequenas modificações nas aulas de Educação Física escolar.

Chiodera et al. (2008), não verificaram aumento na flexibilidade avaliada por meio do Teste de "sentar-e-alcançar" após 64 aulas de Educação Física onde não foi realizada intervenção alguma (32 semanas). Fato semelhante foi encontrado pelo presente estudo com relação ao GCF e GCM. Além dos estudos que verificaram 0 efeito de exercícios de alongamentos durante aulas de Educação Física, outros autores verificaram que a flexibilidade aumenta significativamente após uma intervenção extra-escolar de três e quatro sessões semanais de 15 e 31 semanas, respectivamente (ARDOY et al., 2011; SANTONJA et al., 2007; STEPHENS; WENTZ, 1998).

Por outro lado, parece não existir diferença significativa para a flexibilidade avaliada por meio do Teste de "sentar-e-alcançar" entre crianças e adolescentes que praticam esportes fora da escola, se comparadas com crianças que apenas frequentam aulas de educação física duas vezes por semana. (BEETS; PITETTI, 2005; KOUTEDAKIS; BOUZIOTAS, 2003). Geralmente, exercícios de alongamento são realizados no aquecimento ou ao final da aula de Educação
Física. Esta prática exerce efeito crônico positivo, pois aumenta a flexibilidade de crianças que participam das aulas de Educação Física com frequência de duas vezes na semana (COLEDAM et al., 2012; SANTONJA et al., 2007), fato que pode explicar os resultados semelhantes no desempenho da flexibilidade de crianças e adolescentes que praticam esportes fora da escola comparadas com crianças que apenas frequentam aulas de Educação Física (BEETS; PITETTI, 2005; KOUTEDAKIS; BOUZIOTAS, 2003). Além disso, a inclusão de exercícios de alongamento durante as aulas de Educação Física pode explicar as diferenças nos resultados do presente estudo com os obtidos por CHIODERA et al. (2008), que não foram utilizados exercícios de alongamento, não sendo verificado aumento da flexibilidade após 32 semanas em crianças. Tais resultados indicam que a participação nas aulas de Educação Física, nas quais são realizados exercícios de alongamento, pode proporcionar estímulos suficientes para manutenção ou aumento da flexibilidade, não havendo necessidade de atividades extra-escolares para esta capacidade.

Com relação ao teste de impulsão vertical, a execução de saltos no início das aulas de Educação Física, com frequência de duas vezes por semana e duração de 32 semanas, aumenta significativamente a altura alcançada pelos meninos no teste de impulsão vertical (WEEKS et al., 2008). Da mesma forma, o efeito de intervenções extra-escolares que consistiram de sessões de corrida, saltos, exercícios com bolas e esportes sobre o teste de impulsão vertical e impulsão horizontal foi estudado. De forma geral, ocorreram aumentos significativos no teste de impulsão vertical (BONHAUSER et al., 2005) e impulsão horizontal (MATVIENKO; AHRABIFARD, 2010) por meio de programas de intervenção em relação ao grupo controle, com frequências de três a cinco sessões semanais, com duração de 4 a 8 semanas em crianças. Por outro lado, Stenevi-Lundgren et al. (2009) e (2010), não encontraram diferença significativa no teste de impulsão vertical após o período de intervenção de 48 e 12 semanas respectivamente, com aumento semanal de 200 min. de atividade física em crianças.

A comparação dos resultados deste estudo com os disponíveis na literatura é difícil devido às 
diferentes durações e procedimentos utilizados nos programas de intervenção. $O$ tipo de exercício utilizado é um fator que pode ser limitante em crianças, uma vez que a baixa motivação na execução dos exercícios pode influenciar negativamente os resultados da intervenção proposta em meninas (WEEKS et al., 2008). Além disso, o teste utilizado deve ser específico aos exercícios realizados e ser responsivo aos efeitos da intervenção (ARRUDA et al., 2011).

Este fato pode justificar os resultados obtidos por Stenevi-Lundgren et al. (2009) e (2010), nos quais não foram verificadas diferenças significativas no teste de impulsão Vertical após o programa de intervenção realizado por meio de jogos de basquetebol, handebol, futebol, corridas, escalada e exercícios de ginástica. Apesar de não serem encontradas diferenças significativas no teste de impulsão vertical, foram observados aumentos significativos no torque muscular e na massa corporal magra (Stenevi-Lundgren et al. 2009; 2010). Para uma melhor interpretação dos resultados, em nosso estudo foram utilizados exercícios com cordas que tipicamente são praticados de forma motivada pelas crianças, e 0 teste utilizado foi 0 de impulsão vertical, específico ao movimento de pular corda que é realizado no plano vertical. Além disso, no presente estudo foi descrita a quantificação dos exercícios durante toda a intervenção, o que torna possível a interpretação dos resultados, sua reprodução e aplicação prática dos modelos propostos, porém este não é um procedimento adotado em diversos estudos (MATVIENKO; AHRABI-FARD, 2010; STENEVI-LUNDGREN et al., 2009; STEPHENS; WENTS, 1998). Aspecto importante a ser observado é o fato dos meninos $(\Delta \%=-4,84$ e $-0,58$, diferença média de $-0,14$ ($2,19-1,92)$ e $-1,23(-2,99-0,52)$ e meninas $(\Delta \%$ $=2,62$ e 2,64, diferença média de 0,66 (-0,85$2,18)$ e $0,70(-1,17-2,57) \mathrm{cm}$ dos grupos controles terem apresentado manutenção dos resultados nos testes de "sentar e alcançar" e impulsão vertical respectivamente. Comportamento semelhante foi demonstrado por outros estudos, que encontraram manutenção da flexibilidade em meninos (CHIODERA et al., 2008), e em crianças de ambos os sexos (SAINZ DE BARANDA et al., 2006; RODRÍGUEZ et al., 2008; STEPHENS; Wents, 1998). Da mesma forma, a impulsão vertical se mantém estável em indivíduos integrantes de grupos controle (BONHAUSER et al., 2005; STENEVILUNDGREN et al., 2009; 2010; WEEKS et al., 2008). Tais resultados sugerem que, caso 0 objetivo da aula seja o aumento da flexibilidade e impulsão vertical pode-se realizar exercícios de alongamento e saltos durante as aulas de Educação Física em crianças.

A Educação Física escolar possui como um de seus objetivos é proporcionar níveis adequados de aptidão física relacionada à saúde dos escolares (GUEDES, 1999). Desta forma, a aplicação de programas de intervenção durante aulas de Educação Física devem ter a duração adequada para que haja tempo hábil para aplicação dos demais conteúdos. Em estudos que utilizaram parte do tempo da aula de Educação Física para a aplicação de um programa de intervenção o tempo utilizado variou de 5 a $10 \mathrm{~min}$. apenas para exercícios de alongamento (SAINZ DE BARANDA et al., 2006; RODRÍGUEZ et al., 2008) ou de saltos (GUNTER et al., 2008; WEEKS et al., 2008,). Além disso, diversos pesquisadores utilizaram estratégias após o horário de aula, com frequência de 3 a 5 dias por semana (BEETS; Pitetti, 2005; BONHAUSER et al., 2005; KOUTEDAKIS; BOUZIOTAS, 2003; MATVIENKO; AHRABIFARD, 2010; Stenevi-Lundgren et al., 2009; 2010; STEPHENS; WENTZ, 1998).

Apesar de serem programas eficazes para o aumento do desempenho motor de crianças, a aplicação prática destes achados demanda a contratação de profissionais especializados, disponibilidade de espaços, infra-estrutura para a intervenção e por ser realizado em período oposto ao horário escolar dificulta a aderência dos alunos, o que limita a aplicação destes modelos. Sendo assim, o programa de intervenção realizado por meio de exercícios utilizado no presente estudo demonstra grande aplicação prática, uma vez que o tempo utilizado para a realização do programa de intervenção totalizou $7 \mathrm{~min}$. para exercícios de alongamentos e exercícios utilizando cordas, não alterando o conteúdo da aula. Além disso, os procedimentos utilizados podem ser realizados pelo professor de Educação Física da própria escola e com a participação do aluno durante o horário escolar, o que pode aumentar a aderência ao programa de intervenção. 
Algumas limitações deste estudo devem ser consideradas. Para a flexibilidade foram realizados exercícios de alongamento para outros grupamentos musculares, não sendo possível identificar possíveis alterações em outros segmentos corporais apenas com o teste de "sentar-e-alcançar". Os resultados do teste de "sentar-e-alcançar" são influenciados por outros grupamentos musculares como os eretores da coluna, rotadores do quadril e gastrocnêmio (HOLT et al., 1999), não sendo possível estimar a flexibilidade dos músculos isquiotibiais isoladamente. Com relação à intervenção realizada através exercício com cordas, sabe-se que exercícios envolvendo saltos aumentam o desempenho da impulsão vertical (CHANNELL; BARFIELD, 2008), agilidade (MEYLAN; MALATESTA, 2009) e velocidade (KOTZAMANIDIS, 2006) em crianças e adolescentes. Este estudo utilizou apenas o teste de impulsão vertical, não sendo possível analisar alterações em outras variáveis de desempenho motor.

Como em outros estudos (SANTONJA et al., 2007; RODRÍGUES et al., 2008; WEEKS et al., 2008), foi comparado o efeito do programa de intervenção apenas em dois momentos, antes e após as 12 semanas de intervenção. Este delineamento impede a análise da possibilidade de haver um momento onde houve maior aumento ou platô para a flexibilidade e impulsão vertical ao longo da intervenção.

Outro aspecto a ser apontado quando é analisado o efeito do treinamento físico em crianças e adolescentes é a maturação biológica, que pode influenciar o desenvolvimento das capacidades físicas de forma diferente. É conhecido que o processo de maturação biológica exerce influência aumentando o desempenho de adolescentes nas capacidades que requerem força como o teste de impulsão vertical utilizado no presente estudo (JONES et al., 2000), enquanto que não modifica 0 desempenho da flexibilidade (MINATTO et al., 2010). No presente estudo, a maturação biológica não foi avaliada, no entanto devido a característica homogênea das variáveis analisadas dos grupos controle e intervenção no momento PRÉ, idade média dos participantes (9 anos) e o tempo curto de intervenção (12 semanas) são indicativos de que os resultados obtidos não podem ser atribuídos à maturação biológica.

De acordo com os resultados deste estudo e as evidências disponíveis pode-se afirmar que o programa de intervenção realizado por meio de exercícios foi eficiente em aumentar a flexibilidade e a impulsão vertical de crianças escolares durante as aulas de Educação Física, sem alterar o conteúdo proposto pela escola. Além disso, exercícios de alongamentos e saltos podem ser incluídos durante a aula pelos professores de Educação Física, uma vez que em crianças que não realizam tais exercícios a flexibilidade e a impulsão vertical apenas se mantêm nos meninos e nas meninas. Os exercícios de saltos realizados por meio de cordas parecem ser uma boa opção para aumentar a Impulsão Vertical de crianças escolares, sendo adequado para a faixa etária por serem aceitos e já fazerem parte da cultura desta população. Apesar disso, sugere-se ainda a realização de outros estudos com objetivo de verificar 0 efeito de outros modelos de intervenção, identificar em quais momentos do programa ocorre os aumentos do desempenho, o efeito destes programas sobre outros testes motores e em amostras de populações diferentes da analisada neste estudo.

\section{Conclusão}

Foram verificados aumentos significativos da flexibilidade e Impulsão Vertical nos grupos intervenção masculino e feminino enquanto que os grupos de controle masculino e feminino apresentaram manutenção da flexibilidade e da Impulsão Vertical. Verifica-se que pequenas alterações na elaboração das atividades durante as aulas de Educação Física podem proporcionar melhoras significativas na flexibilidade e impulsão vertical, sugerindo a necessidade de melhor estruturação da aula de Educação Física. $O$ aumento do desempenho da flexibilidade e potência muscular é benéfico principalmente para crianças que não participam de treinamento físico sistematizado e que possuem baixo desempenho nos testes analisados, fato que pode representar risco à saúde.

\section{Referências}

ARDOY, D. N.; FERNANDEZ-RODRIGUEZ, J. M.; RUIZ, J. R.; CHILLÓN, ROMERO, V. E.; CASTILLO, M.; ORTEGA, F. B. Improving 
Physical Fitness in Adolescents Through a School-Based Intervention: the EDUFIT Study. Revista Espanhola de Cardiologia, Madrid, v. 64, n. 6, 2011. Disponível em: $<$ http://apps.elsevier.es/watermark/ctl servlet? $\mathrm{f}=$ 10 \&pident articulo $=90020931$ \&pident usuario $=0$ \&pcontactid=\&pident revista $=255 \&$ ty $=33 \&$ accion =L\&origen=elsevier\&web=www.revespcardiol.org \&lan=en\&fichero=255v64n06a90020931pdf001.p $\underline{\mathrm{df}}>$. Acesso em: 16 set. 2011.

ARRUDA, G. A.; PIANCA, H. J. C.; OLIVEIRA, A. R. Correlação do Teste de 1RM com aspectos maturacionais, neuromotores, antropométricos e a composição corporal em crianças e adolescentes. Revista Brasileira de Medicina do Esporte, São Paulo, v. 17, n. 3, p. 179-183, 2011. Disponível em

$<$ http://www.scielo.br/scielo.php?pid=S151786922011000300006\&script=sci arttext>. Acesso em: 16 set. 2011.

BARBANTI, V. J. Dicionário de educação física e esporte. São Paulo: Manole, 2003.

BEETS, M. W.; PITETTI, K. H. Contribution of physical education and sport to health-related fitness in high school students. The Journal of School Health, Columbus, v. 75, n. 1, p. 25-30, 2005.

BONHAUSER, M.; FERNANDEZ, G.; PÜSCHEL, K.; YAÑEZ, F.; MONTERO, J.; THOMPSON, B.; CORONADO, G. Improving physical fitness and emotional well-being in adolescents of low socioeconomic status in Chile: results of a schoolbased controlled trial. Health Promotion International, Eynsham, v. 20, n. 2, p. 113122, 2005. Disponível em:

<http://heapro.oxfordjournals.org/content/20/2/11 3.full.pdf+html>. Acesso em: 16 set. 2011.

BOVET, P.; AUGUSTE, ROBERT, A.; BURDETTE, H. Strong inverse association between physical fitness and overweight in adolescents: a large school-based survey. International Journal of Behavioral Nutrition and Physical Activity, Londres, v. 24, n. 4, p. 479-486, 2007. Disponível em:

$<$ http://www.ijbnpa.org/content/4/1/24>. Acesso em: 31 mai. 2012.

CASTRO-PIÑERO, J.; ARTERO, E. G.; ESPAÑA-ROMERO, V.; ORTEGA, F. B.; SJÖSTRÖM, M.; SUNI, J.; RUIZ, J. R. Criterionrelated validity of field-based fitness tests in youth: A systematic review. British Journal of Sports Medicine, London, v. 44, n. 13, p. 934943, 2009.

CHANNELL, B. T.; BARFIELD, J. P. Effect of Olympic and traditional resistance training on vertical jump improvement in high school boys.
Journal of Strength and Conditioning

Research, Champaign, v. 22, n. 5, p. 1522-1527, 2008.

CHILLÓN, P et al. Hip flexibility is the main determinant of the back-saver sit-and-reach test in adolescents. Journal of Sports Sciences, London, v. 28, n. 6, p. 641-648, 2010.

CHIODERA, P. et al. Specifically designed physical exercise programs improve children's motor abilities. Scandinavian Journal of Medicine and Science in Sports,

Copenhagen, v. 18, n. 2, p. 179-187, 2008.

Disponível em:

$<$ http://onlinelibrary.wiley.com/doi/10.1111/i.1600$\underline{0838.2007 .00682 . x / p d f}>$ Acesso em 16 set 2011.

COLEDAM, D. H. C.; ARRUDA, G. A.;

OLIVEIRA, A. R. Efeito Crônico do alongamento estático realizado durante o aquecimento sobre a flexibilidade de crianças. Revista Brasileira de Cineantropometria e Desempenho Humano, Florianópolis, v. 14, n. 3, p.296-304, 2012. Disponível em:

$<$ http://www.scielo.br/scielo.php?script=sci arttext \&pid=S1980-

$\underline{00372012000300006 \& \text { Ing }=p t \& n r m=i s o}>$. Acesso em: 31 mai. 2012.

DÓREA, V. et al. Aptidão Física Relacionada à Saúde em Escolares de Jequié, BA, Brasil. Revista Brasileira de Medicina do Esporte, São Paulo, v. 14, n. 6, p. 494-499, 2008.

Disponível em:

$<$ http://www.scielo.br/pdf/rbme/v14n6/a04v14n6.p df $>$. Acesso em: 16 set. 2011.

DUNCAN, M. J.; WOODFIELD, L. A. Acute effects of warm up protocol on flexibility and vertical jump on children. Journal of exercise physiology, Duluth, v. 9, n. 3, p. 9-16, 2006. Disponível em:

http://faculty.css.edu/tboone2/asep/Duncan.doc. Acesso em: 16 set. 2011.

FELDMAN, D. E.; SHRIER, I.; ROSSIGNOL, M.; ABENHAIM, L. Risk Factors for the Development of Low Back Pain in Adolescence. American Journal of Epidemiology, Baltimore, v. 154, n. 1, p. 30-36, 2001. Disponível em: $<$ http://aje.oxfordjournals.org/content/154/1/30.full .pdf+html>. Acesso em: 16 set. 2011.

GUEDES, D. P. Educação para a saúde mediante programas de Educação Física escolar. Motriz. Revista de Educação Física. UNESP, Rio claro, v. 5, n. 1, p. 10-14, 1999. Disponível em:

<http://www.rc.unesp.br/ib/efisica/motriz/05n1/5n1 ART04.pdf>. Acesso em: 16 set. 2011. 
GUEDES, D. P.; GUEDES, E. R. P. Manual prático para avaliação em Educação Física. Barueri: Manole; 2006.

GUEDES, D. P. Implicações associadas ao acompanhamento do desempenho motor de crianças e adolescentes. Revista Brasileira de Educação Física e Esporte, São Paulo, v. 21, p. 37-60, 2007. Disponível em:

$<$ http://www.revistasusp.sibi.usp.br/pdf/rbefe/v21n esp/v21nespa03.pdf>. Acesso em: 16 set. 2011.

GUNTER, K.; BAXTER-JONES, A.

D.; MIRWALD, R. L.; ALMSTEDT, H.; FULLER, A.; DURSKI, S.; SNOW, C. Jump starting skeletal health: a 4-year longitudinal study assessing the effects of jumping on skeletal development in pre and circum pubertal children. Bone, New York, v. 42, n. 4, p. 710-718, 2008.

HOLT, L. E.; PELHAM, T. W.; BURKE, D. G. Modifications to the Standard Sit-and-Reach Flexibility Protocol. Journal of Athletic Training, Dallas, v. 34, n. 1, p. 43-47, 1999. Disponível em: $<$ http://www.ncbi.nlm.nih.gov/pmc/articles/PMC13 22873/pdf/jathtrain00005-0057.pdf Acesso 16 set 2011>. Acesso em: 31 mai. 2012

JONES, M. A.; HITCHEN, P. J.; STRATTON, G. The importance of considering biological maturity when assessing physical fitness measures in girls and boys aged 10 to 16 years. Annals of Human Biology, Londres, v. 27, n. 1, p. 57-65, 2000.

JONES, M. A.; STRATTON, G.; REILLY, T.; UNNITHAN, V. B. Biological risk indicators for recurrent non-specific low back pain in adolescents. British Journal of Sports

Medicine, London, v. 39, n. 3, p. 137-140, 2005. Disponível em:

$<$ http://www.ncbi.nlm.nih.gov/pmc/articles/PMC17 25147/pdf/v039p00137.pdf>. Acesso em: 16 set. 2011.

KOTZAMANIDIS, C. Effect of plyometric training on running performance and vertical jumping in prepubertal boys. Journal of Strength and Conditioning Research, Champaign, v. 20, n. 2, p. 441-445, 2006.

KOUTEDAKIS, Y.; BOUZIOTAS, C. National physical education curriculum: motor and cardiovascular health related fitness in Greek adolescents. British Journal of Sports

Medicine, London, v. 37, n. 4, p. 311-314, 2003.

Disponível em:

<http://www.ncbi.nlm.nih.gov/pmc/articles/PMC17 24688/pdf/v037p00311.pdf>. Acesso em: 16 set. 2011.

MATVIENKO, O.; AHRABI-FARD, I. The effects of a 4-week after-school program on motor skills and fitness of kindergarten and first-grade students. American Journal of Health

Promotion, Lawrence, v. 24, n. 5, p. 299303, 2010.

MEREDITH, M.D. Parental overview of Fitnessgram Assessment. Dallas. The Cooper Institute for Aerobics Research, 2008.

MEYLAN, C.; MALATESTA, D. Effects of inseason plyometric training within soccer practice on explosive actions of young players. Journal of Strength and Conditioning Research, Champaign, v. 23, n. 9, p. 2605-2613, 2009.

MIKKELSSON, L. O.; NUPPONEN, H.; KAPRIO, J.; KAUTIAINEN, H.; MIKKELSSON, M.;

KUJALA, U. M. Adolescent flexibility, endurance strength, and physical activity as predictors of adult tension neck, low back pain, and knee injury: a 25 year follow up study. British Journal of Sports Medicine, London, v. 40, p. 107-113, 2006. Disponível em:

$<$ http://www.ncbi.nlm.nih.gov/pmc/articles/PMC24 92014/pdf/107.pdf >. Acesso em: 16 set. 2011.

MINATTO, G.; RIBEIRO, R. R.; ACHOUR JUNIOR, A.; SANTOS, K. D. Idade, maturação sexual, variáveis antropométricas e composição corporal: influências na flexibilidade. Revista Brasileira de Cinentropometria e Desempenho Humano, Florianópolis, v. 12, n. 3, p. 152-158, 2010. Disponível em:

<http://www.rbcdh.ufsc.br/DownloadArtigo.do?arti go=564 > . Acesso em: 31 mai. 2012.

PADILLA-MOLEDO, C.; RUIZ, J. R.; ORTEGA, F. B.; MORA, J.; CASTRO-PIÑERO, J. Associations of muscular fitness with psychological positive health, health complaints, and health risk behaviors in Spanish children and adolescents. Journal of Strength and

Conditioning Research, Champaign, v. 26, n. 1, p. $167-173,2012$.

PETERSON, M. D.; ALVAR, B. A.; RHEA, M. R. The contribution of maximal force production to explosive movement among young collegiate athletes. Journal of Strength and Conditioning Research, Champaign, v. 20, n. 4, p. 867873, 2006.

RODRÍGUEZ, P. L.; SANTONJA, F. M.; LÓPEZMIÑARRO, P. A.; BARANDA, P. S.; YUSTE, J. L. Effect of physical education stretching programme on sit-and-reach score in schoolchildren. Science and Sports, Paris, v. 23, p. 170-175, 2008.

RONQUE, E. R. V.; CYRINO, E. S.; DÓREA, V.; SERASSUELO JÚNIOR, H.; GALDI, E. H. G.; ARRUDA, M. Diagnóstico da aptidão física em escolares de alto nível socioeconômico: avaliação referenciada por critérios de saúde. 
Revista Brasileira de Medicina do Esporte, São Paulo, v. 13, n. 2, p. 71-76, 2007. Disponível em:

$<$ http://www.scielo.br/pdf/rbme/v13n2/01.pdf > . Acesso em: 16 set. 2011.

RUIZ, J. R.; ORTEGA, F. B.; WÄRNBERG, J.; MORENO, L. A.; CARRERO, J. J.; GONZALEZGROSS, M.; et al. Inflammatory Proteins and Muscle Strength in Adolescents: The AVENA Study. Archives of pediatrics and adolescent medicine, Chicago, v. 162, n. 5, p. 462-468, 2008.

SAINZ DE BARANDA, P. S.; RODRÍGUES, P. L.; SANTONJA, F. M.; LÓPEZ, P. A.; ANDÚJAR, P.; FERRER, V.; PASTOR, A. Effect of hamstring stretching exercises on the toe-touch test in elementary school children. Journal of Human Movement Studies, London, v. 51, p. 277-289, 2006.

SANTONJA, F. M.; BARANDA S. A. P.; RODRÍGUEZ , P. L.; MIÑARRO, P. A. L.; JORDANA, C. M. Effects of frequency of static stretching on straight-leg raise in elementary school children. The Journal of Sports Medicine and Physical Fitness, Torino, v. 47, n. 3, p. 304-308, 2007.

STEENE-JOHANNESSEN, J.; ANDERSSEN, S. A.; KOLLE, E.; ANDERSEN, L. B. Low Muscle Fitness Is Associated with Metabolic Risk in Youth. Medicine and Science in Sports and Exercise, Hagerstown, v. 41, n. 7, p. 1361-1367, 2009.

STENEVI-LUNDGREN, S.; DALY, R. M.; LINDÉN, C.; GÄRDSELL, P.; KARLSSON, M. K. Effects of a daily school based physical activity intervention program on muscle development in prepubertal girls. European Journal of Applied Physiology, Berlim, v. 105, n. 4, p. 533-

541, 2009.

STENEVI-LUNDGREN, S.; DALY, R. M.; KARLSSON, M. K. A school-based exercise intervention program increases muscle strength in prepubertal boys. International journal of pediatrics, Cairo, 2010. Disponível em:

$<$ http://www.ncbi.nlm.nih.gov/pmc/articles/PMC29 05710/pdf/lJPED2010-307063.pdf>. Acesso em:

16 set. 2011.

STEPHENS, M. B.; WENTZ, S. W. Supplemental fitness activities and fitness in urban elementary school classrooms. Family Medicine, Kansas, v. 30, n. 3, p. 220-223, 1998.

VESCOVI, J. D.; MCGUIGAN, M. R.

Relationships between sprinting, agility, and jump ability in female athletes. Journal of Sports Sciences, London, v. 26, n. 1, p. 97-107, 2008.
WEEKS, B. K; YOUNG, C. M.; BECK, B. R. Eight months of regular in-school jumping improves indices of bone strength in adolescent boys and Girls: the POWER PE study. Journal of Bone and Mineral Research, Washington, v. 23, n. 7, p. 1002-1011, 2008. doi: http://dx.doi.org/10.1359/JBMR.080226

\section{Endereço:}

Diogo Henrique Constantino Coledam Rua Delaine Negro, 90, Jardim Alto da Colina Londrina PR Brasil

86051-980

Telefone: (43) 9653-3513

e-mail: diogohcc@yahoo.com.br

Recebido em: 16 de setembro de 2011.

Aceito em: 22 de agosto de 2012.

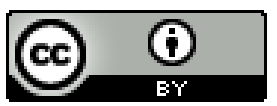

Motriz. Revista de Educação Física. UNESP, Rio Claro, SP, Brasil - elSSN: 1980-6574 - está licenciada sob Creative Commons - Atribuiç̣ão 3.0 